\title{
TAFAMIDIS FOR THE TREATMENT OF TRANSTHYRETIN AMYLOIDOSIS
}

Massimiliano Lorenzini ${ }^{1,2}$, Perry M Elliott ${ }^{1}$.

${ }^{1}$ University College London Institute for Cardiovascular Science and Barts Heart Centre, St.

Bartholomew's Hospital, London, United Kingdom.

${ }^{2}$ Cardiology, Department of Experimental, Diagnostic and Specialty Medicine, Alma Mater Studiorum University of Bologna, Italy.

Word count: 2590

Corresponding author:

Professor Perry M Elliott

UCL Institute for Cardiovascular Science

Paul O'Gorman Building, 72 Huntley Street,

London WC1E 6DD,

United Kingdom

perry.elliott@ucl.ac.uk 


\begin{abstract}
Transthyretin (TTR)-related cardiomyopathy is an underdiagnosed cause of heart failure but is increasingly recognised in various settings - from patients admitted with heart failure to symptomatic aortic stenosis - and is rapidly becoming the most frequent form of systemic amyloidosis. Following the recent publication of the landmark ATTR-ACT trial, that showed tafamidis to be the first treatment to improve survival in patients with TTR-related cardiac amyloidosis and heart failure, we reviewed the drug's rationale, characteristics and evidence supporting its use in TTR amyloidosis.
\end{abstract}

\title{
KEYWORDS
}

Tafamidis, amyloidosis, transthyretin, cardiomyopathy. 


\section{Introduction}

TTR amyloidosis (ATTR) is one of the two most frequent forms of systemic amyloidosis, the second being primary light chain (AL)-related disease, that has a different pathogenesis, natural history and treatment [1]. ATTR can be hereditary (ATTRm) or acquired (wild-type or ATTRwt) with phenotypes that include familial amylodotic neuropathy (FAP), cardiac amyloidosis and mixed phenotypes. Ocular, renal and meningeal involvement are infrequent [2]. Tafamidis is the first disease-modifying drug approved for the treatment of FAP and following the publication of the landmark ATTR-ACT trial [3], is now a potential therapy for TTR-related cardiomyopathy. In this article we review the drug's characteristics and the evidence supporting its use in ATTR.

\section{Transthyretin structure, function and fibrillogenesis}

Transthyretin (TTR) is a protein encoded by a single gene (TTR) and is synthetized almost exclusively by the liver (minimal amounts being produced by the retina and choroid plexus) [4]. TTR circulates predominantly as a homotetramer, along with a very small amount of dissociated monomers [5]. Each monomer comprises 127 amino acids arranged as 8 antiparallel $\beta$-pleated-sheet domains [6] and the tetramer has two distinct dimer-dimer interfaces, the less stable of the two constituting the highly preserved thyroxine (T4)-binding site [5]. Circulating TTR transports retinol (and retinol-binding protein) and T4. While TTR is the only protein to transport retinol, circulating T4 is mainly transported by albumin or thyroidbinding globulin and $<10 \%$ is transported by TTR [7]. On the other hand, TTR is the primary T4 transporter in cerebrospinal fluid [8].

\section{Pathogenesis of TTR amyloidosis}

Amyloidogenesis begins with the rate-limiting dissociation of the TTR tetramer into dimers, that rapidly dissociate into folded monomers. Partial monomer unfolding through a thermodynamically favourable downhill process then leads to the formation of soluble oligomers and polymerisation into amyloid fibrils $[5,9,10]$ (Figure 1). The extracellular deposition of these insoluble fibrils consisting of misfolded TTR with a $\beta$-sheet conformation, leads to disruption of tissue structure and function. A cytotoxic effect of misfolded TTR monomers and small aggregates may also be important in pathogenesis [11]. 
Both wild-type and mutated TTR can form amyloid deposits, suggesting that the protein is innately prone to form $\beta$-pleated-sheet fibrils [12], but TTR mutations destabilise the TTR tetramer and in some cases accelerate the rate of dissociation [13]. Age-related oxidative stress has also been shown to destabilise TTR tetramers and has been suggested as one mechanism underlying the development of acquired (wild-type) TTR amyloidosis [14].

\section{Clinical Phenotypes in ATTR Amyloidosis}

More than 120 amyloidogenic TTR mutations have been described, all inherited as autosomal dominant traits with incomplete penetrance $[6,15]$. The most common TTR mutation is V30M, that is endemic in parts of Portugal, Sweden and Japan and typically leads to a FAP phenotype [16-18]. A number of TTR variants that cause an exclusively cardiac phenotype have been described [19] including V122I, that has a high prevalence (3.4\%) in AfricanAmericans, but appears to have a low penetrance [20]. Other than genotype [6,16-19,21,22] geographic area, endemic vs non-endemic disease [23,24], sex [25], sex of transmitting parent [16-18] and fibril type (full-length or fragmented) [26] all influence the phenotype. In general, ATTRwt affects the elderly population, with a male predominance (80\%) and is characterised the presence of cardiomyopathy in absence of significant extracardiac involvement, with the exception of carpal tunnel syndrome in $1 / 3$ of patients $[27,28]$.

Neurological involvement in ATTR leads to a sensorimotor polyneuropathy, that starts in the lower extremities as small-fibre dysfunction and progressively ascends. Paraesthesiae and dysaesthesiae are prominent, with or without pain, while motor involvement only occurs in the more advanced phases of the disease [6]. Autonomic dysfunction is frequent, and leads to dyshidrosis, bowel dysfunction (diarrhoea alternating with constipation and varying degrees of weight loss and malnutrition), orthostatic hypotension, urinary retention or incontinence and erectile dysfunction [6,21]. Central nervous system involvement is rare, but leptomeningeal amyloid deposition is described in specific genotypes [6].

The spectrum of cardiac involvement in ATTR ranges from asymptomatic conduction disease to severe cardiomyopathy with refractory heart failure. Infiltration of the conduction system can lead to bundle-branch block, sino-atrial or A-V block. Myocardial infiltration leads to a progressive left ventricular (LV) hypertrophy that can be concentric or asymmetric in distribution [29]. Diastolic dysfunction is prominent with moderate or severe diastolic dysfunction present around $80 \%$ of patients [30]. LV ejection fraction is typically normal or 
mildly impaired but significant impairment of longitudinal function is constantly present, with a typical sparing of the apex [30]. LV stroke volume is often reduced even with a normal ejection fraction, due to a combination of small cavity size and diastolic dysfunction[31]. Thickening of the interatrial septum and A-V valves are often present (albeit rarely with functional consequences), as are small pericardial effusions [30].

\section{Treatment of TTR Amyloidosis}

Until recently the major disease modifying treatment was orthotopic liver transplantation (OLT) for patients with mATTR based on the rationale that TTR is almost exclusively produced by the liver. OLT has been shown to slow or halt neurological disease progression [32] but is limited by perioperative mortality up to $10 \%$ [33] and significant morbidity as well as the consequences of long-term immunosuppression. A further important limitation to the use of OLT is that cardiomyopathy can be a contraindication (unless a combined heart-liver transplant is considered) as cardiac deposits of mutated TTR attract circulating wild-type TTR with a seeding mechanism [34] that leads to a progression of the cardiomyopathy despite the absence of circulating mutant TTR [35].

\section{Tafamidis rationale and mechanism of action}

In Portugal, where the V30M mutation responsible for a typical FAP phenotype is endemic, some patients were noted to have an uncharacteristically mild phenotype. When genotyped, they were found to be compound heterozygotes for the V30M mutation and another TTR variant, T119M [36]. Subsequent studies showed that the T119M variant stabilises the TTR tetramer [37], mitigating the effect of pathogenic mutations and leading to a milder phenotype [38]. Circulating TTR tetramers are stabilised by binding to retinol or T4 [39], but approximately half of the circulating TTR is not bound to a ligand. These observations suggested that a molecule that could stabilise the TTR tetramer could lead to a clinical benefit in ATTR patients.

Tafamidis is a benzoxazole carboxylic acid with no non-steroidal anti-inflammatory activity (unlike other TTR stabilisers such as Diflunisal [40]), that binds selectively and with a high affinity to the T4-binding site of TTR and bridges adjacent dimers [41]. It has been shown to be a dose-dependent kinetic stabiliser of the weaker dimer-dimer interface of TTR tetramer 
under denaturing conditions in vitro and ex vivo, in wild-type TTR and a number of different mutations [41].

Circulating tafamidis is $99.9 \%$ bound to plasma proteins. When taken in fasting conditions, it is rapidly absorbed, reaching peak plasma concentration in 2 hours; administration with food reduces the rate, but not the extent of absorption. Mean half-life is approximately 59 hours and a steady-state is reached after 14 days with a $20 \mathrm{mg}$ once a day regimen [42].

There appears to be no induction or inhibition of tafamidis metabolism and it is probably metabolised by glucuronidation. Patients with moderate liver dysfunction (Child-Pugh score 7-9) have a decreased systemic exposure due to higher fraction of unbound circulating drug, however, these patients have lower levels of circulating TTR and no dose adjustment is necessary. No dose adjustment is required in renal impairment or elderly patients [42].

\section{Clinical trials: neuropathy}

The Fx-005 study [43] was an 18-month randomised double-blind trial comparing tafamidis $20 \mathrm{mg}$ OD to placebo in 128 early stage V30M patients. The co-primary endpoints were a combination of response to treatment (defined as no appreciable increase in the Neuropathy Impairment Score-Lower Limbs (NIS-LL) score from baseline) and change in a self-reported quality of life questionnaire. In the intention to treat analysis, treatment with tafamidis did not demonstrate a significant benefit in terms of NIS-LL response to treatment ( $45.3 \%$ vs $29.5 \%$; $\mathrm{p}=0.068$ ) or quality of life. This was probably due to the a higher than expected drop-out rate for OLT $(21 \%)$ in both treatment arms, since these patients were all defined non-responders. In a prespecified efficacy-evaluable population analysis (that considered the patients who completed the 18-months protocol in the assigned treatment arm), more patients treated with tafamidis were NIS-LL responders $(60.0 \%$ vs $38.1 \% ; \mathrm{p}=0.041)$ and had a better-preserved quality of life. Secondary endpoints also favoured tafamidis, since it was associated with less neurologic progression, muscle weakness and small fibre function deterioration. Modified body mass index (BMI*serum albumin, mBMI), a known prognostic factor in FAP [44], improved in patients who received active treatment and worsened in the placebo arm. The trial also documented a good safety profile for tafamidis as adverse events similar in the two treatment groups.

Further evidence to support the use of tafamidis in FAP comes from the 12-month single treatment open-label extension study of Fx-005 [45]. This found that patients who continued 
on tafamidis maintained a slower disease progression and in patients who had previously received placebo, the rate of disease progression declined, but those who had started treatment earlier had a greater benefit. Another small single treatment open-label study suggested that the benefits of tafamidis treatment extend to non-V30M amyloidosis [46]. The study included 8 different genotypes, all with some degree of cardiac involvement, and found that tafamidis appeared to stabilise quality of life, mBMI, NT-proBNP and echocardiographic parameters. Interim data from the long-term open label extension that includes patients from Fx-005 and the non-V30M open-label study seem to confirm these findings up to 6 years [47] and are consistent with the results from other small open-label single treatment studies $[48,49]$.

Using data from the Transthyretin Amyloidosis Outcomes Survey (THAOS), 252 patients with a predominantly neurological phenotype $(>90 \% \mathrm{~V} 30 \mathrm{M})$ who were treated with tafamidis were compared to matched, untreated patients up to 2 years from baseline. The analysis showed less neurological progression in treated patients but could not demonstrate any difference in survival or time-to-disease progression, probably due to the low number of events [50].

\section{Clinical trials: cardiomyopathy}

Initial studies of tafamidis in cardiac amyloidosis were not encouraging. A phase II open label study investigating tafamidis in 35 patients with cardiac ATTR (mainly wild-type), despite evidence of TTR stabilisation, half the patients showed signs of clinical progression such as heart failure admission, syncope or atrial fibrillation [51]. However, a post-hoc analysis that compared this cohort to patients from the TRACS registry [52] suggested a better survival in patients treated with tafamidis [53]. Nevertheless, it must be noted that the study has limitations in design and the TRACS patients used as historical controls were more severely affected at baseline.

ATTR-ACT [3] was a phase III, three-arm, parallel design, placebo-controlled, double-blind, randomized study that compared tafamidis 80 or $20 \mathrm{mg}$ once daily or matching placebo with a 2:1:2 ratio. To be eligible patients needed an invasive or non-invasive diagnosis of TTRrelated cardiac amyloidosis (defined as interventricular septal thickness $>12 \mathrm{~mm}$ on echo) and evidence of heart failure with a prior hospitalisation or signs or symptoms requiring diuretic treatment with NT-proBNP $>600 \mathrm{pg} / \mathrm{ml}$ and 6-minute walk test distance $>100$ meters. 
Important exclusion criteria included NYHA class IV, severe chronic kidney disease $\left(\mathrm{GFR}<25 \mathrm{~mL} / \mathrm{min} / 1.73 \mathrm{~m}^{2}\right)$, severe malnutrition $\left(\mathrm{mBMI}<600 \mathrm{~kg} / \mathrm{m}^{2} \cdot \mathrm{g} / \mathrm{L}\right)$ and prior OLT. Of the 548 patients screened for the study, 441 were enrolled; median age was $75,90 \%$ were male, $76 \%$ had ATTRwt and $81 \%$ were Caucasian. Tafamidis was safe and well tolerated with no differences in discontinuation and side-effects compared to placebo.

To investigate efficacy, along with a conventional Cox regression, the authors used a novel statistical approach (Finkelstein-Schoenfeld method [54]), conceived to improve the analysis of composite endpoints. This method considers the hierarchical importance of the endpoint components (death > hospitalisation), as well as optimizing data analysis by including all the endpoints met by a patient during follow up (e.g. 2 hospitalisations followed by death), and not only the first as occurs in a Cox regression. For this method patients were stratified according to genotype (ATTRm vs ATTRwt) and baseline NYHA class (I/II vs III) and each patient in the treatment group was compared to each patient in the placebo group. For each paired comparison, the patient who reached the death endpoint last is the winner, only if none of the two patients reaches the endpoint is the hospitalisation endpoint analysed with the same method. However, if the patient who does not reach the endpoint has a shorted follow up than the patient who does, then no win is awarded, and the same applies when neither reaches any endpoint. The trial results can therefore be expressed as a win ratio (total number of treatment group wins / total number of placebo group wins).

In a hierarchical assessment of all-cause mortality followed by cardiovascular (CV)-related hospitalisation, tafamidis (pooled 20 and 80mg treatment) was superior to placebo in the primary analysis (win ratio 1.695, 95\% CI 1.255-2.289; p<0.001). Analysing the endpoint components separately by Cox regression, tafamidis reduced all-cause mortality (78/264, $29.5 \%$ vs. $76 / 177,42.9 \%$; hazard ratio $0.70 ; 95 \%$ CI 0.5-0.96) with survival curves separating around 18 months (Figure 2), but also reduced the rate of CV-related hospitalizations ( 0.48 vs 0.70 hospitalizations per year; relative risk reduction 0.68 ; 95\% CI 0.56-0.81). Treatment effect was confirmed in the prespecified subgroup analysis, with one exception, tafamidis increased CV-related hospitalisations in patients in NYHA class III at baseline. The authors interpreted this finding as due to longer survival in patients treated with tafamidis leading to a higher number on CV-related hospitalisations in patients with a severe cardiomyopathy at baseline.

A positive effect of tafamidis was also confirmed in the secondary outcomes reducing the 
decline in the 6-minute walk test distance a quality of life questionnaire score with differences first visible at 6 months treatment.

\section{Future perspective}

Cardiac amyloidosis is undoubtedly underdiagnosed, but a growing awareness of the condition and the possibility of a non-invasive diagnosis with bone-tracer scintigraphy [55] are changing this. The number of mildly affected patients is therefore destined to grow significantly. The coexistence of ATTR and aortic stenosis is of particular interest and initial data suggest a worse prognosis in this subgroup [56-58], and this issue is being investigated prospectively among elderly patients with severe aortic stenosis being considered for intervention (ATTRact-AS study, NCT03029026).

Despite some concerns from the neuropathy trials, tafamidis was well-tolerated and remarkably safe in ATTR-ACT. It will now become the benchmark for novel treatments in ATTR. The drug however has yet to be licensed for the treatment of cardiomyopathy and trials investigating other TTR stabilisers (Study of AG10 in Amyloid Cardiomyopathy, NCT03458130) and the long-term safety of tafamidis (NCT02791230) are ongoing. Also, a number of questions regarding its use patients with cardiomyopathy remain unanswered including use in asymptomatic patients and aortic stenosis - and will require dedicated prospective trials.

Two recently published phase III trials investigated agents that inhibit the hepatic synthesis of TTR in ATTR polyneuropathy. Patisiran [59] and inotersen [60], both appear to be effective, but patisiran has a better safety profile and may reverse myocardial involvement in the subgroup of patients with cardiomyopathy [61]. Further evidence from dedicated cardiomyopathy trials is needed. Future research may also address the possibility of combination treatment with a stabilising agent such as tafamidis and inhibitors of TTR synthesis. 


\section{FIGURE CAPTIONS}

Figure 1: Transthyretin (TTR) amyloidogenesis is blocked by tafamidis-mediated stabilization of tetrameric TTR. Reproduced with permission from Coelho et al [43].

Figure 2: Tafamidis reduced all-cause mortality in the ATTR-ACT trial. Reproduced with permission from Maurer et al [3]. 


\section{EXECUTIVE SUMMARY}

\section{Transthyretin structure, function and fibrillogenesis}

- Transthyretin (TTR) is a protein encoded by a single gene (TTR) and is synthetized almost exclusively by the liver.

- TTR circulates predominantly as a homotetramer and transports retinol and thyroxine (T4). It is the only protein to transport retinol, as well as $<10 \%$ of circulating T4.

\section{Pathogenesis of TTR amyloidosis}

- The dissociation of the TTR tetramer into dimers is the rate-limiting step of amyloidogenesis.

- From monomers, the protein unfolds through a thermodynamically favourable downhill process leading to the formation of soluble oligomers and polymerisation into amyloid fibrils with a $\beta$-sheet conformation.

- Along with disruption of tissue structure and function, a cytotoxic effect of misfolded TTR monomers and small aggregates may also be important.

- TTR is innately prone to form $\beta$-pleated-sheet fibrils but TTR mutations and agerelated oxidative stress have been shown to destabilise the TTR tetramer.

\section{Clinical Phenotypes in TTR Amyloidosis}

- $\quad T T R$ mutations are inherited as autosomal dominant traits with incomplete penetrance.

- In hereditary TTR-related amyloidosis (ATTRm), phenotype is related to genotype, sex, geographic area, endemic vs non-endemic disease, sex of transmitting parent and fibril type (full-length or fragmented).

- The acquired form of the disease (wild-type or ATTRwt) affects the elderly, with a male predominance (80\%) and is characterised by cardiomyopathy in absence of significant extracardiac involvement.

- Neurological involvement leads to a sensorimotor polyneuropathy, with a prominent sensory component, motor involvement in the advanced phases and frequent autonomic dysfunction.

- Cardiac involvement leads to conduction disease and a progressive left ventricular hypertrophy, with prominent diastolic dysfunction and a typically normal or mildly impaired ejection fraction. 


\section{Treatment of TTR Amyloidosis}

- Orthotopic liver transplantation (OLT) slows or halts neurological disease progression in ATTRm but is limited by perioperative mortality and morbidity, long-term immunosuppression, and importantly cardiomyopathy can be a contraindication.

\section{Tafamidis rationale and mechanism of action}

- A specific TTR variant stabilises the TTR tetramer and is responsible for a milder phenotype in some ATTRm patients by mitigating the effect of pathogenic mutations.

- TTR tetramers are stabilised by binding to retinol or T4 and approximately half of the circulating TTR is not bound to a ligand.

- Tafamidis binds selectively and with a high affinity to the T4-binding site of TTR and bridges adjacent dimers. It is a dose-dependent stabiliser of the weaker dimer-dimer interface in vitro and ex vivo.

\section{Clinical trials: neuropathy}

- In an 18-month randomised double-blind trial comparing tafamidis to placebo in 128 early stage with neurological ATTRm due to the V30M mutation, treatment with tafamidis did not demonstrate a significant benefit in terms of a neuropathy score or quality of life.

- This was probably due to the a higher than expected drop-out rate for OLT and in a prespecified efficacy-evaluable population analysis tafamidis increased the rate of responders according to neuropathy score with a better-preserved quality of life.

- The use of tafamidis in neurological ATTRm is also supported to some degree by data from an open-label extension study, a small single treatment open-label study in nonV30M patients, as well as data from the Transthyretin Amyloidosis Outcomes Survey (THAOS) registry.

\section{Clinical trials: cardiomyopathy}

- $A T T R-A C T$ was a randomized controlled double-blind that compared tafamidis to placebo in 441 patients with TTR-related cardiac amyloidosis and evidence of heart failure.

- Tafamidis was safe, well tolerated, and reduced all-cause mortality with survival curves separating around 18 months (Figure 2), as well as reducing the rate of cardiovascular-related hospitalizations. 


\section{REFERENCES}

1. Wechalekar AD, Gillmore JD, Hawkins PN. Systemic amyloidosis. Lancet. 387(10038), 2641-2654 (2016).

2. Ando Y, Coelho T, Berk JL, et al. Guideline of transthyretin-related hereditary amyloidosis for clinicians. Orphanet J. Rare Dis. 8(1), 1-18 (2013).

3. **Maurer MS, Schwartz JH, Gundapaneni B, et al. Tafamidis Treatment for Patients with Transthyretin Amyloid Cardiomyopathy. N. Engl. J. Med. 379(11), 1007-1016 (2018).

\section{Landmark randomised controlled trial investigating the effect of tafamidis on survival and CV-hospitalisations in TTR-related cardiomyopathy.}

4. Dickson PW, Aldred AR, Marley PD, Tu GF, Howlett GJ, Schreiber G. High prealbumin and transferrin mRNA levels in the choroid plexus of rat brain. Biochem. Biophys. Res. Commun. 127(3), 890-5 (1985).

5. Foss TR, Wiseman RL, Kelly JW. The pathway by which the tetrameric protein transthyretin dissociates. Biochemistry. 44(47), 15525-15533 (2005).

6. Benson MD, Kincaid JC. The molecular biology and clinical features of amyloid neuropathy. Muscle Nerve. 36(4), 411-423 (2007).

7. Bartalena L, Robbins J. Thyroid hormone transport proteins. Clin. Lab. Med. 13(3), 583-98 (1993).

8. Schreiber G, Southwell BR, Richardson SJ. Hormone delivery systems to the brain transthyretin. Exp. Clin. Endocrinol. Diabetes. 103(2), 75-80 (1995).

9. Johnson SM, Wiseman RL, Sekijima Y, Green NS, Adamski-Werner SL, Kelly JW. Native state kinetic stabilization as a strategy to ameliorate protein misfolding diseases: A focus on the transthyretin amyloidoses. Acc. Chem. Res. 38(12), 911-921 (2005).

10. Hurshman AR, White JT, Powers ET, Kelly JW. Transthyretin aggregation under partially denaturing conditions is a downhill polymerization. Biochemistry. 43(23), 7365-7381 (2004). 
11. Reixach N, Deechongkit S, Jiang X, Kelly JW, Buxbaum JN. Tissue damage in the amyloidoses: Transthyretin monomers and nonnative oligomers are the major cytotoxic species in tissue culture. Proc. Natl. Acad. Sci. U. S. A. 101(9), 2817-2822 (2004).

12. Hou X, Aguilar M-I, Small DH. Transthyretin and familial amyloidotic polyneuropathy. FEBS J. 274(7), 1637-1650 (2007).

13. Hammarstrom P, Jiang X, Hurshman AR, Powers ET, Kelly JW. Sequence-dependent denaturation energetics: A major determinant in amyloid disease diversity. Proc. Natl. Acad. Sci. 99(Supplement 4), 16427-16432 (2002).

14. Zhao L, Buxbaum JN, Reixach N. Age-related oxidative modifications of transthyretin modulate its amyloidogenicity. Biochemistry. 52(11), 1913-1926 (2013).

15. Mutations In Hereditary Amyloidosis - Mutations in Transthyretin Gene (TTR) [Internet]. http://amyloidosismutations.com/mut-attr.php.

16. Conceição I, De Carvalho M. Clinical variability in type I familial amyloid polyneuropathy (Val30Met): comparison between late- and early-onset cases in Portugal. Muscle Nerve. 35(1), 116-8 (2007).

17. Ikeda S, Nakazato M, Ando Y, Sobue G. Familial transthyretin-type amyloid polyneuropathy in Japan: clinical and genetic heterogeneity. Neurology. 58(7), 1001-7 (2002).

18. Suhr OB, Svendsen IH, Andersson R, Danielsson A, Holmgren G, Ranløv PJ. Hereditary transthyretin amyloidosis from a Scandinavian perspective. J. Intern. Med. 254(3), 225-35 (2003).

19. Rapezzi C, Quarta CC, Obici L, et al. Disease profile and differential diagnosis of hereditary transthyretin-related amyloidosis with exclusively cardiac phenotype: An Italian perspective. Eur. Heart J. 34(7), 520-528 (2013).

20. Quarta CC, Buxbaum JN, Shah AM, et al. The Amyloidogenic V122I Transthyretin Variant in Elderly Black Americans. N. Engl. J. Med. 372(1), 21-29 (2015).

21. Ando Y, Nakamura M, Araki S. Transthyretin-Related Familial Amyloidotic Polyneuropathy. Arch. Neurol. 62(7), 1057 (2005). 
22. Sattianayagam PT, Hahn AF, Whelan CJ, et al. Cardiac phenotype and clinical outcome of familial amyloid polyneuropathy associated with transthyretin alanine 60 variant. Eur. Heart J. 33(9), 1120-7 (2012).

23. Koike H, Misu K, Ikeda S, et al. Type I (transthyretin Met30) familial amyloid polyneuropathy in Japan: early- vs late-onset form. Arch. Neurol. 59(11), 1771-6 (2002).

24. Koike H, Tanaka F, Hashimoto R, et al. Natural history of transthyretin Val30Met familial amyloid polyneuropathy: analysis of late-onset cases from non-endemic areas. J. Neurol. Neurosurg. Psychiatry. 83(2), 152-8 (2012).

25. Rapezzi C, Riva L, Quarta CC, et al. Gender-related risk of myocardial involvement in systemic amyloidosis. Amyloid. 15(1), 40-8 (2008).

26. Suhr OB, Lundgren E, Westermark P. One mutation, two distinct disease variants: unravelling the impact of transthyretin amyloid fibril composition. J. Intern. Med. 281(4), 337-347 (2017).

27. González-López E, Gagliardi C, Dominguez F, et al. Clinical characteristics of wildtype transthyretin cardiac amyloidosis: Disproving myths. Eur. Heart J. 38(24), 18951904 (2017).

28. Connors LH, Sam F, Skinner M, et al. Heart Failure Resulting from Age-Related Cardiac Amyloid Disease Associated with Wild-Type Transthyretin: A Prospective, Observational Cohort Study. Circulation. 133(3), 282-290 (2016).

29. Martinez-Naharro A, Treibel T, Abdel-Gadir A, et al. Magnetic Resonance in Transthyretin Cardiac Amyloidosis. J Am Coll Cardiol. 70(4) (2017).

30. Quarta CC, Solomon SD, Uraizee I, et al. Left ventricular structure and function in transthyretin-related versus light-chain cardiac amyloidosis. Circulation. 129(18), 1840-1849 (2014).

31. Rapezzi C, Quarta CC, Riva L, et al. Transthyretin-related amyloidoses and the heart: a clinical overview. Nat. Rev. Cardiol. 7(7), 398-408 (2010).

32. de Carvalho M, Conceição I, Bentes C, Luís MLS. Long-term quantitative evaluation of liver transplantation in familial amyloid polyneuropathy (Portuguese V30M). 
Amyloid. 9(2), 126-33 (2002).

33. Okamoto S, Wixner J, Obayashi K, et al. Liver transplantation for familial amyloidotic polyneuropathy: Impact on Swedish patients' survival. Liver Transplant. 15(10), 1229-1235 (2009).

34. Saelices L, Chung K, Lee JH, et al. Amyloid seeding of transthyretin by ex vivo cardiac fibrils and its inhibition. Proc. Natl. Acad. Sci. 115(29), E6741-E6750 (2018).

35. Stangou AJ, Hawkins PN, Heaton ND, et al. Progressive cardiac amyloidosis following liver transplantation for familial amyloid polyneuropathy: implications for amyloid fibrillogenesis. Transplantation. 66(2), 229-33 (1998).

36. Coelho T, Chorão R, Sousa A, Alves I, Torres MF, Saraiva MJM. Compound heterozygotes of transthyretin Met30 and transthyretin Met119 are protected from the devastating effects of familial amyloid polyneuropathy. Neuromuscul. Disord. 6, S20 (1996).

37. Longo Alves I, Hays MT, Saraiva MJM. Comparative stability and clearance of [Met30]transthyretin and [Met119]transthyretin. Eur. J. Biochem. 249(3), 662-668 (1997).

38. Hammarström P, Wiseman RL, Powers ET, Kelly JW. Prevention of transthyretin arnyloid disease by changing protein misfolding energetics. Science (80-. ). 299(5607), 713-716 (2003).

39. White JT, Kelly JW. Support for the multigenic hypothesis of amyloidosis: the binding stoichiometry of retinol-binding protein, vitamin A, and thyroid hormone influences transthyretin amyloidogenicity in vitro. Proc. Natl. Acad. Sci. U. S. A. 98(23), 13019$24(2001)$.

40. Sekijima Y, Dendle MA, Kelly JW. Orally administered diflunisal stabilizes transthyretin against dissociation required for amyloidogenesis. Amyloid. 13(4), 23649 (2006).

41. *Bulawa CE, Connelly S, Devit M, et al. Tafamidis, a potent and selective transthyretin kinetic stabilizer that inhibits the amyloid cascade. Proc. Natl. Acad. Sci. U. S. A. 109(24), 9629-34 (2012). 


\section{In vitro and ex vivo evidence of the effect of tafamidis on TTR.}

42. European Medicines Agency summary of the European public assessment report (EPAR) for Vyndaqel.

http://www.ema.europa.eu/ema/index.jsp?curl=pages/medicines/human/medicines/002 294/human_med_001498.jsp\&mid=WC0b01ac058001d124.

43. *Coelho T, Maia LF, Martins da Silva, A Waddington Cruz, M Plante-Bordeneuve, V Lozeron $\mathrm{P}$, et al. Tafamidis for transthyretin familial amyloid polyneuropathy: a randomized, controlled trial. Neurology. 79(8), 785-792 (2012).

\section{Randomised controled trial of tafamidis for the treatment of TTR-related neuropathy in patients with V30M mutation}

44. Suhr O, Danielsson A, Holmgren G, Steen L. Malnutrition and gastrointestinal dysfunction as prognostic factors for survival in familial amyloidotic polyneuropathy. J. Intern. Med. 235(5), 479-85 (1994).

45. Coelho T, Maia LF, Da Silva AM, et al. Long-term effects of tafamidis for the treatment of transthyretin familial amyloid polyneuropathy. J. Neurol. 260(11), 2802$2814(2013)$.

46. Merlini G, Planté-Bordeneuve V, Judge DP, et al. Effects of tafamidis on transthyretin stabilization and clinical outcomes in patients with non-Val30Met transthyretin amyloidosis. J. Cardiovasc. Transl. Res. 6(6), 1011-1020 (2013).

47. Barroso FA, Judge DP, Ebede B, et al. Long-term safety and efficacy of tafamidis for the treatment of hereditary transthyretin amyloid polyneuropathy: results up to 6 years. Amyloid. 24(3), 194-204 (2017).

48. Yukio A, Yoshiki S, Konen O, et al. Effects of tafamidis treatment on transthyretin (TTR) stabilization, efficacy, and safety in Japanese patients with familial amyloid polyneuropathy (TTR-FAP) with Val30Met and non-Varl30Met: A phase III, openlabel study. J. Neurol. Sci. 362, 266-271 (2016).

49. Conceição I, Miranda B, Castro J, de Carvalho M. Hereditary amyloidosis related to transthyretin V30M: disease progression in treated and untreated patients. Eur. J. Neurol. , Epub ahead of print (2018). 
50. Mundayat R, Stewart M, Alvir J, et al. Positive Effectiveness of Tafamidis in Delaying Disease Progression in Transthyretin Familial Amyloid Polyneuropathy up to 2 Years: An Analysis from the Transthyretin Amyloidosis Outcomes Survey (THAOS). Neurol. Ther. 7, 87-101 (2018).

51. Maurer MS, Grogan DR, Judge DP, et al. Tafamidis in Transthyretin Amyloid Cardiomyopathy: Effects on Transthyretin Stabilization and Clinical Outcomes. Circ. Hear. Fail. 8(3), 519-526 (2015).

52. Ruberg FL, Maurer MS, Judge DP, et al. Prospective evaluation of the morbidity and mortality of wild-type and V122I mutant transthyretin amyloid cardiomyopathy: The Transthyretin Amyloidosis Cardiac Study (TRACS). Am. Heart J. 164(2), 222-228.e1 (2012).

53. Sultan MB, Gundapaneni B, Schumacher J, Schwartz JH. Treatment With Tafamidis Slows Disease Progression in Early-Stage Transthyretin Cardiomyopathy. Clin. Med. Insights Cardiol. 11, 1-4 (2017).

54. *Pocock SJ, Ariti CA, Collier TJ, Wang D. The win ratio: A new approach to the analysis of composite endpoints in clinical trials based on clinical priorities. Eur. Heart J. 33(2), 176-182 (2012).

Clear explanation of a novel method to optimize the analys of composite endpoints in clinical trials

55. $* *$ Gillmore JD, Maurer MS, Falk RH, et al. Nonbiopsy diagnosis of cardiac transthyretin amyloidosis. Circulation. 133(24), 2404-2412 (2016).

Multicenter study that provided the evidence basis for reaching a definitive diagnosis of TTR-related cardiac amyloidosis non-invasively.

56. Longhi S, Lorenzini M, Gagliardi C, et al. Coexistence of Degenerative Aortic Stenosis and Wild-Type Transthyretin-Related Cardiac Amyloidosis. JACC Cardiovasc. Imaging. 9(3), 325-327 (2016).

57. Castano A, Narotsky DL, Hamid N, et al. Unveiling transthyretin cardiac amyloidosis and its predictors among elderly patients with severe aortic stenosis undergoing transcatheter aortic valve replacement. Eur. Heart J. 38(38), 2879-2887 (2017). 
58. Cavalcante JL, Rijal S, Abdelkarim I, et al. Cardiac amyloidosis is prevalent in older patients with aortic stenosis and carries worse prognosis. J. Cardiovasc. Magn. Reson. 19(1), 98 (2017).

59. Adams D, Gonzalez-Duarte A, O'Riordan WD, et al. Patisiran, an RNAi Therapeutic, for Hereditary Transthyretin Amyloidosis. N. Engl. J. Med. 379(1), 11-21 (2018).

60. Benson MD, Waddington-Cruz M, Berk JL, et al. Inotersen Treatment for Patients with Hereditary Transthyretin Amyloidosis. N. Engl. J. Med. 379(1), 22-31 (2018).

61. Solomon SD, Adams D, Kristen A, et al. Effects of Patisiran, an RNA Interference Therapeutic , on Cardiac Parameters in Patients with Hereditary TransthyretinMediated Amyloidosis : An Analysis of the APOLLO Study. Circulation. (Epub ahead of print) (2018). 